TRANSACTIONS OF THE

AMERICAN MATHEMATICAL SOCIETY

Volume 353, Number 11, Pages 4673-4686

S 0002-9947(01)02828-8

Article electronically published on April 24, 2001

\title{
GENERIC FINITENESS FOR DZIOBEK CONFIGURATIONS
}

\author{
RICHARD MOECKEL
}

\begin{abstract}
The goal of this paper is to show that for almost all choices of $n$ masses, $m_{i}$, there are only finitely many central configurations of the Newtonian $n$-body problem for which the bodies span a space of dimension $n-2$ (such a central configuration is called a Dziobek configuration). The result applies in particular to two-dimensional configurations of four bodies and threedimensional configurations of five bodies.
\end{abstract}

\section{INTRODUCTION}

Consider the Newtonian $n$-body problem in $d$-dimensional space:

$$
\ddot{x}_{i}=\sum_{j \neq i} \frac{m_{j}\left(x_{j}-x_{i}\right)}{r_{i j}^{3}}, \quad i=1, \ldots, n .
$$

Here $m_{i}$ are the masses of the bodies, $x_{i} \in \mathbf{R}^{d}$ are their positions, and $r_{i j}=\left|x_{i}-x_{j}\right|$ are their mutual distances. The vector $x=\left(x_{1}, \ldots, x_{n}\right) \in \mathbf{R}^{d n}$ will be called the configuration of the system. For the differential equations to be well-defined we require that the configuration be nonsingular, that is, $r_{i j} \neq 0$ for $i \neq j$.

Although the problem is set in $\mathbf{R}^{d}$, a given configuration $x$ may not span this space. Define the dimension, $\delta(x)$, of a configuration $x$ to be the dimension of the affine hull of $x$, that is, the dimension of the smallest affine subspace of $\mathbf{R}^{d}$ which contains all of the points $x_{i}$. Configurations with $\delta(x)=1,2,3$ will be called collinear, planar and spatial, respectively.

Let

$$
M=m_{1}+\cdots+m_{n}, \quad c=\frac{1}{M}\left(m_{1} x_{1}+\cdots+m_{n} x_{n}\right),
$$

be the total mass and center of mass of the bodies. A configuration $x$ is called a central configuration if the acceleration vectors of the bodies satisfy

$$
\sum_{j \neq i} \frac{m_{j}\left(x_{j}-x_{i}\right)}{r_{i j}^{3}}+\lambda\left(x_{i}-c\right)=0, \quad i=1, \ldots, n,
$$

for some constant $\lambda$. Such configurations give rise to simple, explicit solutions of the $n$-body problem [7. If the bodies are placed in a central configuration and released with zero initial velocity, they will collapse homothetically to a collision at c. If the central configuration is planar, one can also choose initial velocities which lead to a periodic solution for which the configuration rigidly rotates around $c$ with angular velocity $\sqrt{\lambda}$.

Received by the editors December 29, 2000.

1991 Mathematics Subject Classification. Primary 70F10, 70F15, 37N05.

Key words and phrases. Celestial mechanics, central configurations, $n$-body problem.

(C)2001 American Mathematical Society 
The dimension of any nonsingular configuration of $n \geq 2$ bodies lies in the range $1 \leq \delta(x) \leq n-1$. A collinear central configuration is called a Moulton configuration after F.R. Moulton who proved that for a fixed mass vector $m=\left(m_{1}, \ldots, m_{n}\right)$ and a fixed ordering of the bodies along the line, there exists a unique collinear central configuration (up to translation and scaling) 12]. At the other extreme of the dimension range, Lagrange showed that when $n=3$, the only central configuration with $\delta(x)=n-1=2$ is the equilateral triangle, and it is a central configuration for all choices of the masses [6]. An analogous result holds for all $n$. For example, the only spatial central configuration of $n=4$ bodies is the regular tetrahedron, which is central for all choices of the masses [8].

Unfortunately, for other values of the dimension the problem of finding or even counting the central configurations is very difficult. The dimension $\delta(x)=2$ is undoubtedly the most interesting of all, because planar central configurations give rise to physically realistic periodic orbits. For $n=4$, Otto Dziobek formulated the planar central configuration problem in terms of mutual distances $r_{i j}$ and obtained algebraic equations characterizing the central configurations 3. His approach has been adopted and developed by Albouy in his study of the central configurations with four equal masses [1]. The proper generalization to higher $n$ is not the planar problem but rather the case $\delta(x)=n-2$. Following [2] we call such a central configuration a Dziobek configuration. For configurations with $\delta(x)=n-2$, the mutual distances are subject to a single constraint, the vanishing of the CayleyMenger determinant. Lower dimensions seem much more complicated from this point of view.

The main goal of this paper is to study the question of finiteness - given $n$ masses, is the number of central configurations finite? Here it will be shown that the number of Dziobek configurations is finite, at least for generic choices of the masses. More precisely, there is a polynomial in the masses, $P\left(m_{1}, \ldots, m_{n}\right)$, such that if $P(m) \neq 0$ then the number of Dziobek configurations is finite and does not exceed a certain bound which depends only on $n$. For the planar four-body problem, a proof of this was given in [11]; see also [5]. The proof given here is different and generalizes to higher $n$. The result for the spatial five-body problem is new as are the more exotic results for higher $n$. Before trying to prove finiteness it is worth noting that at least one Dziobek configuration exists for each $n$. Just put $n-1$ equal masses at the vertices of a regular $(n-2)$-simplex and an arbitrary $n$-th mass at the barycenter.

Acknowledgments. Thanks to Alain Albouy, Alain Chenciner and Rui Fernandes for their hospitality and to the Équipe d'Astronomie et Systèmes Dynamiques at the Bureau des Longitudes, the Université de Paris VII, the Institut Superior Técnico, and the School of Mathematics at the University of Minnesota for their support.

\section{Geometry of Configurations}

In this section we will present some elementary facts about the geometry of configurations of $n$ points in $\mathbf{R}^{d}$. Since the dimension of a configuration always satisfies $\delta(x) \leq n-1$ there is no loss of generality in taking $d=n-1$.

Let $x=\left(x_{1}, \ldots, x_{n}\right)$ with $x_{i} \in \mathbf{R}^{n-1}$. We associate with $x$ an $n \times n$ matrix and its determinant: 


$$
X=\left[\begin{array}{lll}
1 & \cdots & 1 \\
\hline x_{1} & \cdots & x_{n}
\end{array}\right], \quad \omega(x)=|X| .
$$

Then it is easy to see that

$$
\delta(x)=\operatorname{rank} X-1 .
$$

In particular, $\delta(x)=n-1$ if and only if $\omega(x) \neq 0$. This also follows from the fact that $\omega(x)$ is $(n-1)$ ! times the oriented volume of the $(n-1)$-simplex spanned by $x$.

We have $\delta(x) \leq n-2$ if and only if there is a nonzero vector $\Delta=\left(\Delta_{1}, \ldots, \Delta_{n}\right)$ in the kernel of $X$, that is,

$$
\begin{gathered}
\Delta_{1}+\cdots+\Delta_{n}=0, \\
x_{1} \Delta_{1}+\cdots+x_{n} \Delta_{n}=0 .
\end{gathered}
$$

Moreover $\delta(x)=n-2$ if and only if $\Delta$ is unique up to a constant factor.

When $\delta(x) \leq n-2$ we may assume without loss of generality that $x_{i} \in \mathbf{R}^{n-2}$. In this case there is a nice formula for a vector $\Delta$ satisfying (5). The matrix $X$ is now $(n-1) \times n$. Augment $X$ by adding a row of zeros along the bottom to obtain an $n \times n$ matrix $\tilde{X}$ (this amounts to viewing the $x_{i}$ as vectors in $\mathbf{R}^{n-1}$ with vanishing last coordinate). Let $\hat{x}_{k}=\left(x_{1}, \ldots, \widehat{x_{k}}, \ldots, x_{n}\right)$ denote the configuration of $(n-1)$ bodies where the $k$-th position vector has been deleted and let $\hat{X}_{k}$ be the $(n-1) \times(n-1)$ matrix analogous to (4) which is clearly obtained from $X$ by deleting the $k$-th column. Then up to a common sign factor, the quantities

$$
\Delta_{k}=(-1)^{k+1}\left|\hat{X}_{k}\right|
$$

are the cofactors of the zero entries in the last row of $\tilde{X}$. Since $|\tilde{X}|=0$, a familiar property of determinants shows that the vector $\Delta=\left(\Delta_{1}, \ldots, \Delta_{n}\right)$ is in the kernel of $\tilde{X}$ and so satisfies (5). Up to a factor of $(n-2)$ !,

$$
\omega\left(\hat{x}_{k}\right)=\left|\hat{X}_{k}\right|
$$

gives the oriented $(n-2)$-volume spanned by $\hat{x}_{k}$. Therefore we have $\delta(x)=n-2$ if and only if at least one of these determinants is nonzero. For later use, we note that $\omega\left(\hat{x}_{k}\right)$ can be found from the $(n-2)$-fold wedge product:

$$
(-1)^{\tau}\left(x_{1}-x_{j}\right) \wedge \cdots \wedge\left(x_{n}-x_{j}\right)=\omega\left(\hat{x}_{k}\right) e_{1} \wedge \cdots \wedge e_{n-2}
$$

where the factor $\left(x_{k}-x_{j}\right)$ is omitted, $j$ is any index with $j \neq k, e_{i}$ are the standard basis vectors in $\mathbf{R}^{n-2}$ and $\tau(j, k)$ is $(-1)^{j+1}$ if $j<k$ and $(-1)^{j}$ if $j>k$.

Next we will reformulate the dimension criteria above in terms of the mutual distances $r_{i j}=\left|x_{i}-x_{j}\right|$ or rather, their squares $s_{i j}=\left|x_{i}-x_{j}\right|^{2}$. When working with these quantities we will regard the $p=\frac{1}{2} n(n-1)$ distances with $1 \leq i<j \leq n$ as independent variables, the others being obtained by transposing the subscripts. Let $s \in \mathbf{R}^{p}$ be the vector with components $s_{i j}, 1 \leq i<j \leq n$.

Using equations (5) we have

$$
\sum_{j} s_{i j} \Delta_{j}=\left|x_{i}\right|^{2} \sum_{j} \Delta_{j}-2 x_{i} \cdot \sum_{j} x_{j} \Delta_{j}+\sum_{j}\left|x_{j}\right|^{2} \Delta_{j}=\sum_{j}\left|x_{j}\right|^{2} \Delta_{j}
$$


where $i$ is any fixed index and the sum over $j$ runs from 1 to $n$ (here $s_{i i}=0$ ). The result is independent of $i$ and we denote it by $-\Delta_{0}$. Define the Cayley-Menger matrix and determinant by

$$
A(s)=\left[\begin{array}{cccccc}
0 & 1 & 1 & 1 & \ldots & 1 \\
1 & 0 & s_{12} & s_{13} & \ldots & s_{1 n} \\
1 & s_{12} & 0 & s_{23} & \ldots & s_{2 n} \\
1 & s_{13} & s_{23} & 0 & \ldots & s_{3 n} \\
\vdots & \vdots & \vdots & \vdots & & \vdots \\
1 & s_{1 n} & s_{2 n} & s_{3 n} & \ldots & 0
\end{array}\right], \quad F(s)=|A(s)| .
$$

Then we have $A(s) \Delta=0$ where now $\Delta=\left(\Delta_{0}, \Delta_{1}, \ldots, \Delta_{n}\right)$.

The next result expresses the dimension of a configuration in terms of $F$ and its cofactors. We will use the notation $F_{i j}$ for the cofactor of the element in the $i$-th row and $j$-th column of $F$.

Proposition 1. Let $x$ be a configuration of $n$ bodies and let $F$ be the associated Cayley-Menger determinant. Then $\delta(x) \leq n-2$ if and only if $F=0$. If $F=0$, the following are equivalent:

i. $\delta(x)=n-2$,

ii. at least two of the principal cofactors $F_{i i}$ are nonzero,

iii. $\operatorname{rank} A=n$.

Proof. Assume without loss of generality that $x_{i} \in \mathbf{R}^{n-1}$. If $\delta(x) \leq n-2$, then equations (5) have a nonzero solution $\left(\Delta_{1}, \ldots, \Delta_{n}\right)$. The first equation implies that at least two of the $\Delta_{i}, 1 \leq i \leq n$, are nonzero. Defining $-\Delta_{0}$ by the sums (8) as above and setting $\Delta=\left(\Delta_{0}, \Delta_{1}, \ldots, \Delta_{n}\right)$ we have $A(s) \Delta=0$. It follows that $F(s)=|A(s)|=0$.

Conversely, if $F(s)=0$ the equation $A(s) \Delta=0$ has a nonzero solution $\Delta$. For these $\Delta_{i}$, the sums (8) are all equal to $-\Delta_{0}$ and also $\sum_{j} \Delta_{j}=0$, where the sums all run from 1 to $n$ as before. It follows that all the position vectors $x_{i}$ satisfy $\alpha \cdot x_{i}=\beta$ where

$$
\alpha=2 \sum_{j} x_{j} \Delta_{j}, \quad \beta=\Delta_{0}+\sum_{j}\left|x_{j}\right|^{2} \Delta_{j} .
$$

If $\alpha \neq 0$ this is the equation of a hyperplane we have $\delta(x) \leq n-2$ as required. On the other hand if $\alpha=0$, then equations (5) hold, so $\delta(x) \leq n-2$ in this case as well.

Next suppose $F=0$. If $\delta(x)=n-2$, then for any $j \in\{1, \ldots, n\}$ the vectors $x_{i}-x_{j}, 1 \leq i \leq n, i \neq j$, span a vector space of dimension $n-2$. So we can delete one of them, say the $i$-th, and still span the space. Then $\delta\left(\hat{x}_{i}\right)=n-2$ and so $F_{i i} \neq 0$. Moreover, since $i \neq j$ where $j$ was an arbitrary index, at least two of these cofactors are nonzero. Thus i implies ii.

It is clear that ii implies iii. If iii holds then the nonzero vector in the kernel of $A$ is unique up to a constant multiple. By a familiar result from determinant theory, every row of the matrix of cofactors $F_{i j}$ solves these equations. Therefore all of the rows are proportional to $\Delta$ and hence also to one another. This is expressed by the quadratic identities:

$$
F_{i k} F_{j l}=F_{i l} F_{j k} \quad \text { for all } \quad i, j, k, l .
$$


In particular we have $F_{i i} F_{j j}=F_{i j}^{2}$. Now iii implies that at least one cofactor $F_{i j} \neq 0$ and it follows that at least one principal cofactor $F_{i i} \neq 0$. Then $\delta\left(\hat{x}_{i}\right)=n-2$ and this implies i. QED

The next result gives a convenient representation of the cofactors of a CayleyMenger determinant satisfying the conditions in proposition 1. It applies to determinants of the form (9) whether or not they arise from a real configuration of $n$-bodies.

Proposition 2. Let $A(s)$ and $F(s)$ be a matrix and determinant of the form (9) with $s_{i j}$ arbitrary complex numbers. Suppose $F(s)=0$ but at least one of the cofactors $F_{i j} \neq 0$. Then if $\Delta=\left(\Delta_{0}, \Delta_{1}, \ldots, \Delta_{n}\right)$ is any nonzero solution of $A(s) \Delta=0$, there exists a constant $\kappa \neq 0$ such that

$$
F_{i j}=\kappa \Delta_{i} \Delta_{j} .
$$

Moreover, at least two of the $\Delta_{i}, 1 \leq i \leq n$, are nonzero.

Proof. The assumptions imply that $A(s)$ has rank $n$. Therefore the nonzero vector $\Delta$ is unique up to a constant multiple. As in the proof of proposition 1, each row of the matrix of cofactors also satisfies this equation and so is proportional to $\Delta$. In other words $F_{i j}=\kappa_{i} \Delta_{j}$ where $\kappa_{i}$ is some constant. By symmetry we also have $F_{i j}=\kappa_{j} \Delta_{i}$. From $\kappa_{i} \Delta_{j}=\kappa_{j} \Delta_{i}$ and the fact that $\Delta \neq 0$ it follows that $\kappa_{i}=\kappa \Delta_{i}$ for some constant $\kappa$ and (11) follows. Since not all the $F_{i j}$ vanish, we must have $\kappa \neq 0$. Now from the form of the equations, $\Delta_{0}$ cannot be the only nonzero component of $\Delta$. Since the sum of the $\Delta_{j}, 1 \leq j \leq n$, vanishes, at least two of these numbers must be nonzero. QED

Note that under the assumptions of proposition 2, we have $F_{i i} \neq 0$, for at least two values of $i \in\{1, \ldots, n\}$, just as in proposition 1 . Also $F_{i j} \neq 0$ for some pair of indices $1 \leq i<j \leq n$. We also remark that the representation (11) still holds without the assumption $F_{i j} \neq 0$, but if all the $F_{i j}=0$ we also have $\kappa=0$. The quadratic identities (10) follow immediately from (11).

For $n=2$, we have $F\left(s_{12}\right)=2 s_{12}$. This vanishes if and only if the two bodies coincide. Since this causes difficulties with the Newtonian force law, we assume $n \geq 3$ from now on. The case $n=3$ is also special because when we set $s_{i j}=r_{i j}^{2}$ the determinant factorizes as

$$
F(s)=\left(r_{12}-r_{13}-r_{23}\right)\left(r_{13}-r_{12}-r_{23}\right)\left(r_{23}-r_{12}-r_{13}\right)\left(r_{12}+r_{13}+r_{23}\right) .
$$

\section{The Equations for Dziobek Configurations}

In this section, we derive equations for central configurations with $\delta(x)=n-2$ in terms of the mutual distances. Assume without loss of generality that the ambient space has dimension $d=n-2$. Following [15] we set

$$
\lambda=\frac{M}{r_{0}^{3}}
$$

in equation (3), where $M=m_{1}+\cdots+m_{n}$ and $r_{0}$ is a new constant. This makes the equations homogeneous in both the $m$ and $r$-variables. Then collecting terms gives

$$
\sum_{j \neq i} m_{j} S_{i j}\left(x_{i}-x_{j}\right)=0, \quad 1 \leq i \leq n
$$


where

$$
S_{i j}=r_{i j}^{-3}-r_{0}^{-3} .
$$

To eliminate the explicit dependence on $x$ we take wedge products of the $i$-th equation with $(n-3)$-dimensional multivectors of the form

$$
v_{i k l}=\left(x_{1}-x_{i}\right) \wedge \ldots \wedge\left(x_{n}-x_{i}\right)
$$

where $i, k, l$ are any three distinct indices between 1 and $n$ and where the wedge product omits the three factors $\left(x_{i}-x_{i}\right),\left(x_{k}-x_{i}\right)$ and $\left(x_{l}-x_{i}\right)$. The result is

$$
m_{k} S_{i k} \Delta_{l}=m_{l} S_{i l} \Delta_{k} .
$$

This follows from (7) and the definition of $\Delta_{k}$. These equations generally contain less information than equations (13). For configurations with $\delta(x)<n-2$, all of the $\Delta_{i}$ vanish and equations (15) are all trivial. However, as we shall see, they are very useful in the case $\delta(x)=n-2$.

Let $S \in \mathbf{R}^{p}$ be the vector with components $S_{i j}, 1 \leq i<j \leq n$. The next result gives a parametrization of the vectors, $S$, arising from Dziobek configuations.

Proposition 3. Let $x$ be a central configuration of $n$ nonzero masses with $\delta(x)=$ $n-2$, and let $S_{i j}$ be given by (14). Then there are real numbers $z_{i}, 1 \leq i \leq n$, and $k \neq 0$ such that

$$
S_{i j}=k z_{i} z_{j} .
$$

Moreover, at least two of the $z_{i}$ are nonzero.

Proof. Since the masses are nonzero we can define

$$
z_{i}=\frac{\Delta_{i}}{m_{i}}, \quad i=1, \ldots, n,
$$

and then (15) can be written

$$
S_{i k} z_{l}=S_{i l} z_{k} .
$$

If $\delta(x)=n-2$, not all of the variables $z_{i}$ can vanish and it follows that there are constants $k_{i}$ such that $S_{i j}=k_{i} z_{j}$. The symmetry of $S_{i j}$ gives $k_{i} z_{j}=k_{j} z_{i}$ and it follows that $k_{i}=k z_{i}$ for some constant $k$. This proves (16).

To see that $k \neq 0$ note that otherwise we would have $S_{i j}=0$ for all $i \neq j$ and from (14), all the mutual distances would be equal. But then the configuration would be a regular $(n-1)$-simplex with $\delta(x)=n-1$. For the same reason, at least two of the $z_{i}$ are nonzero. QED

From (16) it follows immediately that the vectors, $S$, arising from Dziobek configurations satisfy the quadratic equations

$$
S_{i k} S_{j l}=S_{i l} S_{j k}
$$

where $i, j, k, l$ can be any four distinct indices between 1 and $n$. However, these equations are weaker than (16). For example, when $n=4$ let $S_{12}=S_{13}=S_{23}=0$ and $S_{14}=S_{24}=S_{34}=1$. Then $S$ satisfies (18) but it is not of the form (16). For this reason it is preferable to work with the variables $z_{i}$ instead of the variables $S_{i j}$. 


\section{Some Facts from Algebraic Geometry}

The equations in the last two sections were derived from geometrical considerations and from the equations for central configurations with real, nonzero masses. From this point on, we will be concerned only with proving that these equations have finitely many solutions and for this purpose it is better to allow all the variables to be complex. Then the powerful results of the theory of complex algebraic geometry can be applied. In this section we present some of these results with references to the book of Shafarevich [13.

Let $z=\left(z_{0}, \ldots, z_{n}\right) \in \mathbf{C}^{n+1}, z \neq 0$, and let $[z] \in \mathbf{C P}^{n}$ be the corresponding point in complex projective space. A projective variety or closed projective set is a subset of $\mathbf{C P}^{n}$ of the form

$$
V=\left\{[z]: p_{i}(z)=0, i \in I\right\}
$$

where $I$ is any index set and $p_{i}(z)=p_{i}\left(z_{0}, \ldots, z_{n}\right)$ are homogeneous polynomials. Homogeneity is needed so that the zero set is well-defined on projective space. Any variety can be defined using finitely many polynomials. Projective varieties are the closed sets of a topology on $\mathbf{C P}^{n}$ called the Zariski topology. A Zariski-open set is just the complement of a variety.

A quasi-projective variety is a set of the form $V=X \backslash Y$, where $X$ and $Y$ are projective varieties. Equivalently, $V$ is a Zariski-open subset of a projective variety. This is the most useful concept for our problem because we need to exclude the singular configurations. A quasi-projective variety $V$ can always be completed to get a projective variety, $\bar{V}$, called the projective closure of $V$. This is defined as the smallest projective variety containing $V$.

We will also make use of varieties in a product of projective spaces. A subvariety $V \subset \mathbf{C P}^{n} \times \mathbf{C P}^{k}$ is a set of the form

$$
V=\left\{([z],[w]): p_{i}(z, w)=0, i \in I\right\}
$$

where the $p_{i}(z, w)=p_{i}\left(z_{0}, \ldots, z_{n} ; w_{0}, \ldots, w_{k}\right)$ are separately homogeneous in $z$ and in $w$, possibly with different degrees of homogeneity. One can easily generalize to products of three or more projective spaces.

A subvariety of a quasi-projective variety $W \subset V$ is defined to be a Zariskiclosed subset (this would be called a closed subvariety or just a closed subset in [13]). In other words, $W$ is obtained from $V$ by imposing additional polynomial equations. $V$ is called irreducible, if it cannot be written as a union of two proper subvarieties. It is a basic fact that every variety can be uniquely decomposed as a union of irreducible varieties

$$
V=V_{1} \cup \ldots \cup V_{N}
$$

where $V_{i} \not \subset V_{j}$, for $i \neq j$. The $V_{i}$ are called the irreducible components of $V$.

A proper subvariety of an irreducible variety should be considered a "small" subset. A nonempty Zariski-open set, being the complement of a proper subvariety, should be considered "large". For example, a nonempty Zariski-open subset is open and dense in the usual topology of $\mathbf{C P}^{n}$. We will say that a property holds almost everywhere or generically on an irreducible variety $V$, if it holds on a nonempty Zariski-open subset.

The idea of a regular mapping between varieties will play an important role later on. Let $V \subset \mathbf{C P}^{n}$ and $W \subset \mathbf{C P}^{k}$ be two quasi-projective varieties. A mapping 
$f: V \rightarrow W$ is regular at a point $[\bar{z}] \in V$ if locally near $[\bar{z}]$ it is given by a formula

$$
\left(\zeta_{0}, \ldots, \zeta_{k}\right)=\left(f_{0}\left(z_{0}, \ldots, z_{n}\right), \ldots, f_{k}\left(z_{0}, \ldots, z_{n}\right)\right)
$$

where the $f_{i}(z)$ are homogeneous polynomials of the same degree which do not all vanish at $[\bar{z}]$. The map $f$ is regular if it is regular at every point $[\bar{z}] \in V$. There may or may not be one such formula which satisfies the required conditions globally on $V$. Similarly, we can define regular mappings between varieties living in products of projective spaces. The projections $\pi_{1}: \mathbf{C P}^{n} \times \mathbf{C P}^{k} \rightarrow \mathbf{C P}^{n}$ and $\pi_{2}: \mathbf{C P}^{n} \times \mathbf{C P}^{k} \rightarrow \mathbf{C} \mathbf{P}^{k}$ are regular mappings. For example, in the formula $\pi_{2}(z, w)=\left(w_{0}, \ldots, w_{k}\right)$, the components $w_{i}$ are never simultaneously zero because they are the homogenous coordinates of a point in $\mathbf{C P}^{k}$.

Another useful concept is that of a dominant mapping. A regular mapping $f: V \rightarrow W$ is dominant if its image $f(V)$ is not contained in any proper subvariety of $W$. Equivalently, this means $\overline{f(V)} \supset W$ and also $\overline{f(V)}=\bar{W}$. In this case, it can be shown that there is a Zariski-open subset $W_{0}$ of $W$ with $f(V) \supset W_{0}$ [13, Ch.I.5, Th.6]. If we define $V_{0}=f^{-1}\left(W_{0}\right)$, then we have a surjective map of quasi-projective varieties $f: V_{0} \rightarrow W_{0}$.

If $f: V \rightarrow W$ is dominant and if $V$ is irreducible, then $W$ is also irreducible. To see this note that if $W=W_{1} \cup W_{2}$ where $W_{i} \subset W$ are proper subvarieties, then we would have $V=V_{1} \cup V_{2}$ where $V_{i}=f^{-1}\left(W_{i}\right)$ are also proper subvarietes.

There are several equivalent definitions of dimension for quasi-projective varieties 13, Ch.I.6], 4, Ch.11]. If $V$ is any quasi-projective variety, then $\operatorname{dim} V$ is defined as the maximum of the dimensions of its irreducible components, $V_{i}$. For a nonempty, irreducible quasi-projective variety, $\operatorname{dim} V$ can be defined in any of the following ways:

- $\operatorname{dim} V$ is the transcendence degree of the field of rational functions on $V$,

- $\operatorname{dim} V=d$ if $V$ is defined locally at almost every point by $n-d$ polynomials with independent differentials,

- $\operatorname{dim} V=d$ if almost every linear subspace $L \subset \mathbf{C P}^{n}$ of dimension $n-d$ intersects $W$ in a nonempty finite set,

- $\operatorname{dim} V=d$ if $d$ is the smallest integer such that almost every linear subspace $L \subset \mathbf{C P}^{n}$ of dimension $n-d-1$ does not intersect $V$.

The dimension has various useful properties [13, Ch.I.6]. For example, a quasiprojective variety $V$ has $\operatorname{dim} V=0$ if and only if $V$ is a nonempty finite set. Also, a proper subvariety $V^{\prime} \subset V$ of an irreducible variety $V$ always has $\operatorname{dim} V^{\prime}<\operatorname{dim} V$ (more evidence for the idea that $V^{\prime}$ is small). The dimension of $V$ is equal to the dimension of its projective closure $\bar{V}$. The dimension of any Zariski-open subset of an irreducible variety is equal to the dimension of the whole variety.

We will also need some facts about the behavior of dimension under regular mappings, which we collect together as a proposition. Here and in the rest of the paper, the word finite will be taken to allow the case of the empty set.

Proposition 4. Let $f: V \rightarrow W$ be a regular mapping of quasi-projective varieties.

i. If $f$ is dominant, then $\operatorname{dim} V \geq \operatorname{dim} W$.

ii. If every fiber $f^{-1}([\zeta]),[\zeta] \in W$, is finite, then $\operatorname{dim} V \leq \operatorname{dim} W$. More generally, if every fiber has dimension at most $d$, then $\operatorname{dim} V \leq \operatorname{dim} W+d$.

iii. If $W$ is irreducible and $\operatorname{dim} V \leq \operatorname{dim} W$, then almost every fiber is finite.

Proof. We will base the proofs on theorem 7 of [13, Ch.I.6] which states that if $f: V \rightarrow W$ is a surjective map between irreducible quasi-projective varieties, then 
$\operatorname{dim} V \geq \operatorname{dim} W$. Moreover, every irreducible component of every fiber $f^{-1}([\zeta])$ has dimension at least $\operatorname{dim} V-\operatorname{dim} W$ with equality holding for all [ $\zeta]$ in some nonempty Zariski-open subset of $W$. Throughout the proof we will refer to this as theorem 7 .

To prove i, choose an irreducible component $W_{j} \subset W$ of maximal dimension. Then there is some irreducible component $V_{i}$ such that $f: V_{i} \rightarrow W_{j}$ is dominant. Otherwise $f$ itself would not be dominant. As noted above, there are Zariski-open subsets $V_{i 0}, W_{j 0}$ such that $f: V_{i 0} \rightarrow W_{j 0}$ is surjective and these have the same dimensions as $V_{i}, W_{j}$. By theorem 7 we have $\operatorname{dim} V \geq \operatorname{dim} V_{i} \geq \operatorname{dim} W_{j}=\operatorname{dim} W$.

For ii, take any component $V_{i}$ of $V$. Then there is a component $W_{j}$ of $W$ with $f: V_{i} \rightarrow W_{j}$. It is enough to show that for all components, we have $\operatorname{dim} V_{i} \leq$ $\operatorname{dim} W_{j}+d$. If $f: V_{i} \rightarrow W_{j}$ is dominant, then as in the proof of i we may assume $f$ is actually surjective. By assumption, every component of every fiber has dimension at most $d$ and so theorem 7 shows that $\operatorname{dim} V_{i}-\operatorname{dim} W_{j} \leq d$. If $f$ is not dominant, then there is proper subvariety $W_{j}^{\prime} \subset W_{j}$ with $f: V_{i} \rightarrow W_{j}^{\prime}$ dominant, namely, $W_{j}^{\prime}=\overline{f\left(V_{i}\right)} \cap W_{j}$. In this case, theorem 7 shows that $\operatorname{dim} V_{i} \leq \operatorname{dim} W_{j}^{\prime}+d<$ $\operatorname{dim} W_{j}+d$.

Finally consider iii. It suffices to consider the restrictions to the irreducible components $V_{i}$. If $f: V_{i} \rightarrow W$ is not dominant, then the result is trivial because almost every fiber is empty. If it is dominant, then i shows that $\operatorname{dim} V_{i} \geq \operatorname{dim} W$. Since $\operatorname{dim} V \leq \operatorname{dim} W$ we must have $\operatorname{dim} V_{i}=\operatorname{dim} W$. After first restricting to a surjective map of Zariski-open subsets, we find from theorem 7 that almost every fiber has dimension zero and so is a finite set. QED

\section{The Variety of Dziobek Configurations}

In this section we will use the equations satisfied by Dziobek configurations to define a complex, quasi-projective variety and we will compute its dimension. Let $p=\frac{1}{2} n(n-1)$. Let $r \in \mathbf{C}^{p+1}$ be the vector with components $r_{0}$ and $r_{i j}$, $1 \leq i<j \leq n$. To eliminate the scaling symmetry of the problem it is convenient to work in projective space. We will use the notation $[r] \in \mathbf{C P}^{p}$ for the point with homogeneous coordinates $r$. We will also regard the variables $z_{i}$ of proposition 3 as complex and we will introduce an additional variable $z_{0}$. Then we have $z=$ $\left(z_{0}, z_{1}, \ldots, z_{n}\right) \in \mathbf{C}^{n+1}$ and $[z] \in \mathbf{C P}^{n}$.

Equations (14) and (16) imply that for any $r$ arising from a Dziobek configuration, the following equations have a nonzero solution $z \in \mathbf{C}^{n+1}$ :

$$
\begin{aligned}
r_{i j}^{-3}-r_{0}^{-3} & =z_{i} z_{j}, \\
r_{0}^{-3} & =z_{0}^{2},
\end{aligned}
$$

where we have absorbed the factor of $k$ from (16) into the $z_{i}$ variables, and included a new equation defining $z_{0}$. We will use these equations together with the CayleyMenger determinant to define a variety in $\mathbf{C P}^{p} \times \mathbf{C P}^{n}$.

First define the singular set

$$
\Sigma=\left\{([r],[z]) \in \mathbf{C P}^{p} \times \mathbf{C P}^{n}: z_{0} r_{0} \prod_{i<j} r_{i j}=0\right\} .
$$

On the complement of $\Sigma$, (19) are equivalent to the equations

$$
z_{0}^{2}\left(r_{0}^{3}-r_{i j}^{3}\right)=r_{i j}^{3} z_{i} z_{j}
$$


$1 \leq i<j \leq n$, which are separately homogeneous in $r$ and $z$. Then we can define a quasi-projective variety, $V$, which contains all of the Dziobek configurations:

$$
V=\left\{([r],[z]) \in \mathbf{C P}^{p} \times \mathbf{C P}^{n} \backslash \Sigma: F\left(r_{i j}^{2}\right)=0 \text { and (20) hold }\right\} .
$$

We will also have to work with the subvarieties obtained by setting some of the $z_{i}=0$. Let

$$
V_{k}=\left\{([r],[z]) \in V: z_{k+1}=\cdots=z_{n}=0\right\} .
$$

The following result is crucial for proving the generic finiteness theorem we are after. It shows that the variety $V$ containing the Dziobek configurations has the same dimension as the mass space.

Theorem 1. The variety $V$ has $\operatorname{dim} V=n-1$. More generally, $\operatorname{dim} V_{k}=k-1$, $k \geq 2$.

The proof depends on the following curious result:

Proposition 5. Let $\omega_{i j} \in \mathbf{C}, 0 \leq i<j \leq n$, be third roots of unity. Then

$$
\left|\begin{array}{cccccc}
0 & 1 & 1 & 1 & \ldots & 1 \\
1 & 0 & 2 \omega_{12} & \omega_{13} & \ldots & \omega_{1 n} \\
1 & 2 \omega_{12} & 0 & \omega_{23} & \ldots & \omega_{2 n} \\
1 & \omega_{13} & \omega_{23} & 0 & \ldots & \omega_{3 n} \\
\vdots & \vdots & \vdots & \vdots & & \vdots \\
1 & \omega_{1 n} & \omega_{2 n} & \omega_{3 n} & \ldots & 0
\end{array}\right| \neq 0 .
$$

Proof. The determinant can be expanded as a sum of monomials in the $\omega_{i j}$ with integer coefficients. Each monomial is equal to an integer multiple of $1, \omega$ or $\omega^{2}$ where $\omega=-\frac{1}{2}+\frac{\sqrt{3}}{2} i$. Therefore the determinant is of the form $\alpha+\beta \omega+\gamma \omega^{2}$ where $\alpha, \beta, \gamma$ are integers. An expression of this form vanishes if and only if it is a multiple of the minimal polynomial of $\omega, 1+\omega+\omega^{2}$, that is, if and only if $\alpha=\beta=\gamma$. A necessary condition for this is that $\alpha+\beta+\gamma$ be divisible by 3 . Now the sum $\alpha+\beta+\gamma$ is the value of the determinant with all $\omega_{i j}=1$ which turns out to be $(-1)^{n} 4$. So the determinant cannot vanish. QED

Proof of Theorem 1. Let $\pi_{2}: \mathbf{C P}^{p} \times \mathbf{C} \mathbf{P}^{n} \rightarrow \mathbf{C P}^{n}$ be the projection. The proof for $V$ consists of analyzing the fiber and image of the regular mapping $\pi_{2}: V \rightarrow \mathbf{C P}^{n}$. We will show that the projective closure $W=\overline{\pi_{2}(V)}$ has $\operatorname{dim} W=n-1$. Given any $[z] \in \mathbf{C P}^{n}$, the fiber $\pi_{2}^{-1}([z])$ is obtained by solving equations (19) for $[r]$. Since it is clear from the form of the equations that every fiber is finite, parts $i$ and ii of proposition 4 will give $\operatorname{dim} V=n-1$ as well.

To understand the projection $\pi_{2}(V)$ first note that $[z] \in \pi_{2}(V)$, if and only if equations (19) have a solution $r \in \mathbf{C}^{p+1}$ with all components nonzero and with $F\left(r_{i j}^{2}\right)=0$. Then

$$
g_{i j}=\left(z_{i} z_{j}+z_{0}^{2}\right) r_{i j}^{3}-1=0 .
$$

We will use these equations to eliminate the variables $r_{i j}$ from the Cayley-Menger determinant. Consider $F$ and $g_{12}$ as two polynomials in the variable $r_{12}$ with all the other variables viewed as parameters. Let $G$ be the resultant of these two polynomials with respect to $r_{12}$. The resultant is a polynomial function of the coefficients of the two given polynomials, hence it is a polynomial function of $\left(z_{1} z_{2}+z_{0}^{2}\right)$ and the $r_{i j}$ other than $r_{12}$. It is a property of the resultant that if for a 
certain value of the parameters there exists an $r_{12}$ solving both $F=0$ and $g_{12}=0$, then these parameter values satisfy $G=0$. Conversely, if $G=0$ for a certain value of the parameters and if the leading coefficient $z_{1} z_{2}+z_{0}^{2} \neq 0$, then there will be a nonempty finite set of values of $r_{12}$ such that $F=0$ and $g_{12}=0$ both hold.

Continuing to eliminate the $r_{i j}$ in this way we eventually obtain a polynomial $H(z)$ with the property that if for given value of $z$, there exist $r_{i j}$ satisfying both $F=0$ and all of the equations $g_{i j}=0$, then $H(z)=0$. Conversely, if $z$ is any solution of $H(z)=0$ such that all of the quantities $z_{i} z_{j}+z_{0}^{2} \neq 0$, then there is a nonempty finite set of values of the $r_{i j}$ for which $F=0$ and $g_{i j}=0$ hold. Further properties of the resultant imply that the polynomial $H(z)$ is homogeneous.

These computations are too complicated to carry out explicitly except when $n=3$. In that case, we can eliminate $r_{12}, r_{13}, r_{23}$ from each of the factors of (12) separately using a computer algebra system. Each factor leads to a homogeneous polynomial of degree 36 . For example, the last factor leads to an expression of the form $-\left(9 z_{0}^{8} z_{1}^{2} z_{2}^{2}+\ldots\right)^{3}$ where the parentheses contains 50 terms, all homogeneous of degree 12 .

Using $H(z)$ we define a projective variety

$$
W^{\prime}=\left\{[z] \in \mathbf{C P}^{n}: H(z)=0\right\} .
$$

The properties of the resultant mentioned above imply that $\pi_{2}(V) \subset W^{\prime}$. To characterize the image more precisely, recall that the only obstruction to solving for the variables $r_{i j}$ given $z$ is for one of the equations $z_{i} z_{j}+z_{0}^{2}=0$ to hold. Also, if $z_{0}=0$, we cannot solve for $r_{0}$. Define a homogeneous polynomial

$$
K(z)=z_{0} \cdot \prod_{i<j}\left(z_{i} z_{j}+z_{0}^{2}\right)
$$

and a projective variety

$$
B=\left\{[z] \in \mathbf{C P}^{n}: K(z)=0\right\} .
$$

Then $\pi_{2}(V)=W^{\prime} \backslash B$. Some of the irreducible components of $W^{\prime}$ may be entirely contained in $B$. We ignore these and let $W$ be the union of all the other components of $W^{\prime}$. Then $B$ intersects each component of $W$ in a proper subvariety, so $\pi_{2}: V \rightarrow$ $W$ is a dominant mapping. In other words, $W=\overline{\pi_{2}(V)}$. The variety $W^{\prime}$ is defined by a single polynomial equation, $H(z)=0$, in $\mathbf{C P}^{n}$. If we can show that $H(z)$ is not identically zero, then it follows from [13, ChI.6, Th.2], that every irreducible component of $W^{\prime}$ has dimension $n-1$. In particular, we will have $\operatorname{dim} W=n-1$, completing the proof.

To show that $H(z)$ is not identically zero, it suffices to find some $z$ for which the quantities $z_{i} z_{j}+z_{0}^{2}$ are all nonzero but for which the corresponding equations $F=0$ and $g_{i j}=0$ have no solution. Since $H(z)$ is the resultant of these equations, we will have $H(z) \neq 0$. This is where we need proposition 5. Take $z_{0}=1$ and $z_{i}=0,3 \leq i \leq n$. Then for $3 \leq i, j \leq n$ we have $z_{i} z_{j}+z_{0}^{2}=1$ and the equations $g_{i j}=0$ reduce to $r_{i j}^{3}=1$. So these $r_{i j}$ and their squares $s_{i j}$ are all third roots of unity. On the other hand, if we choose $z_{1}, z_{2}$ so that $z_{1} z_{2}+z_{0}^{2}=1 / \sqrt{8}$ then $r_{12}^{3}=\sqrt{8}$ and $s_{12}$ is twice a third root of unity. The Cayley-Menger determinant is exactly as in proposition 5 so $F\left(r_{i j}^{2}\right) \neq 0$.

The proof for $V_{k}$ is similar, but we use the projection $\pi_{2}: V_{k} \rightarrow \mathbf{C P}^{k}$ where we view $\mathbf{C P}^{k}$ as the subset of $\mathbf{C} \mathbf{P}^{n}$ with $z_{k+1}=\cdots=z_{n}=0$. Again we need to see that the resultant $H(z)$ does not vanish identically on $\mathbf{C P}^{k}$. This follows because 
the point $z$ with $H(z) \neq 0$ which we constructed above is actually in $\mathbf{C P}^{k}, k \geq 2$. QED

\section{The Mass Correspondence and Generic Finiteness}

In this section we will prove the generic finiteness theorem. In the last section we studied the variety of Dziobek configurations using the variables $[r] \in \mathbf{C P}^{p},[z] \in$ $\mathbf{C P}^{n}$. Here we also study the mass vector $m=\left(m_{1}, \ldots, m_{n}\right) \in \mathbf{C}^{n},[m] \in \mathbf{C P}^{n-1}$. The equations (15) provide a relation between the masses and the variables $S_{i j}, \Delta_{i}$. Multiplying these equations by $\Delta_{j}$ and using (11) and (14) we find

$$
m_{k} z_{i} z_{k} F_{j l}=m_{l} z_{i} z_{l} F_{j k} .
$$

Here $i, k, l$ must be distinct indices between 1 and $n$, but $j$ is arbitrary. As cofactors of the Cayley-Menger determinant, the quantities $F_{i j}$ are homogeneous polynomials in $r$. Equations (21) are separately homogeneous in $r, z, m$ and we can use them to define a variety in $\mathbf{C} \mathbf{P}^{p} \times \mathbf{C} \mathbf{P}^{n} \times \mathbf{C P}^{n-1}$ :

$$
\begin{aligned}
\Gamma & =\left\{([r],[z],[m]) \in\left(\mathbf{C P}^{p} \times \mathbf{C P}^{n} \backslash \Sigma\right) \times \mathbf{C P}^{n-1}: F\left(r_{i j}^{2}\right)=0 \text { and }(20),(21) \text { hold }\right\} \\
& =\left\{([r],[z],[m]) \in V \times \mathbf{C P}^{n-1}:(21) \text { hold }\right\} .
\end{aligned}
$$

We will make use of the projections $\pi_{12}: \Gamma \rightarrow \mathbf{C P}^{p} \times \mathbf{C P}^{n}$ and $\pi_{3}: \Gamma \rightarrow \mathbf{C P}^{n-1}$.

Decompose $\Gamma$ into irreducible components $\Gamma_{\alpha}$. We will introduce some terminology to describe the behavior of the quantities $z_{i}, F_{i j}$ and $m_{i}$ on these components. Throughout this section will write $f \equiv 0$ if a function $f$ vanishes identically on the component $\Gamma_{\alpha}$ and $f \not \equiv 0$ if it does not vanish identically.

Call $\Gamma_{\alpha}$ a Dziobek component if the following conditions hold:

i. at least two of the $z_{i} \not \equiv 0,1 \leq i \leq n$,

ii. at least two of the principal cofactors $F_{i i} \not \equiv 0,1 \leq i \leq n$.

The non-Dziobek components, if any, are irrelevant for us because they contain no real configurations of dimension $n-2$. To see this note that if $\Gamma_{\alpha}$ does contain some point which arises from a real configuration with $\delta(x)=n-2$ then proposition 3 shows that i must hold and proposition 1 shows that ii must hold.

The next result shows that the vanishing of a cofactor $F_{i j}$ is associated with the vanishing of one of the masses or one of the $z$-variables.

Proposition 6. Let $\Gamma_{\alpha}$ be a Dziobek component and suppose that $F_{i l} \equiv 0$ on $\Gamma_{\alpha}$ for some indices with $1 \leq i, l \leq n$. Then also $m_{i} m_{l} z_{i} z_{l} \equiv 0$ on $\Gamma_{\alpha}$.

Proof. Since $F_{i l} \equiv 0$ equations (10) reduce to $F_{i k} F_{j l} \equiv 0$. If some $F_{i k} \not \equiv 0,0 \leq k \leq$ $n$, then we have $F_{j l} \equiv 0,0 \leq j \leq n$. So either all the $F_{i k}$ vanish identically or all the $F_{j l}$ do. Assume without loss of generality that $F_{j l} \equiv 0,0 \leq j \leq n$.

Since $\Gamma_{\alpha}$ is a Dziobek component, there are at least two indices $k$ such that $F_{k k} \not \equiv$ 0 . Since $F_{l l} \equiv 0$ we may take $k \neq i, l$. Now (21) with $j=k$ gives $m_{l} z_{i} z_{l} F_{k k} \equiv 0$. It follows that $m_{l} z_{i} z_{l} \equiv 0$ and hence also $m_{i} m_{l} z_{i} z_{l} \equiv 0$. QED

A component $\Gamma_{\alpha}$ will be called mass-dominant if $\pi_{3}\left(\Gamma_{\alpha}\right)$ is not contained in any proper subvariety of the mass space, that is, the projective closure satisfies $\overline{\pi_{3}\left(\Gamma_{\alpha}\right)}=\mathbf{C P}^{n-1}$. We can restrict attention to such components because we are only interested in generic masses. It should be noted that if $\Gamma_{\alpha}$ is not mass dominant, then $\pi_{3}\left(\Gamma_{\alpha}\right) \cap \mathbf{R P}^{n-1}$ is also contained in a proper subvariety of the real mass space. To see this, let $f$ be a nonzero complex polynomial which vanishes on $\pi_{3}\left(\Gamma_{\alpha}\right)$. Then the real and imaginary parts of $f$ are real polynomials which vanish 
on $\pi_{3}\left(\Gamma_{\alpha}\right) \cap \mathbf{R P}^{n-1}$. They are also nonzero since any polynomial which vanishes identically on $\mathbf{R P}^{n-1}$ also vanishes identically on $\mathbf{C P}^{n-1}$.

Theorem 2. Any mass-dominant Dziobek component, $\Gamma_{\alpha}$, has $\operatorname{dim} \Gamma_{\alpha}=n-1$.

Proof. By mass dominance, we have $\operatorname{dim} \Gamma_{\alpha} \geq n-1$. It remains to show $\operatorname{dim} \Gamma_{\alpha} \leq$ $n-1$. Call $\Gamma_{\alpha}$ nondegenerate if $z_{i} \not \equiv 0$ and $F_{i j} \not \equiv 0$ for all $1 \leq i, j \leq n$. If $\Gamma_{\alpha}$ is nondegenerate, then it has a nonempty Zariski-open subset, $U_{\alpha}$, on which $z_{i} \neq 0$ and $F_{i j} \neq 0$ for all $1 \leq i, j \leq n$. The projection $\pi_{12}: U_{\alpha} \rightarrow V$ is a regular mapping. All of the fibers, $\pi_{12}^{-1}([r],[z])$, are finite because on $U_{\alpha}$, equations $(21)$ determine the mass ratios uniquely. Now by theorem 1 , the range $V \operatorname{has} \operatorname{dim} V=n-1$. It follows from part ii of proposition 4 that $\operatorname{dim} \Gamma_{\alpha} \leq n-1$ as required.

Proposition 6 shows that every degenerate component has $z_{i} \equiv 0$ for one or more indices $1 \leq i \leq n$. On the other hand, every Dziobek component has $z_{i} \not \equiv 0$ for at least two indices, $i$. Without loss of generality, we may assume that there is some index $2 \leq k<n$ such that $z_{i} \not \equiv 0$ for $1 \leq i \leq k$ and $z_{i} \equiv 0$ for $k+1 \leq i \leq n$.

It follows from proposition 6 that for such a component, $F_{i j} \not \equiv 0$ for $1 \leq i, j \leq k$. On the Zariski-open subset where $z_{i} \neq 0$ and $F_{i j} \neq 0,1 \leq i, j \leq k$, we can solve (21) uniquely for the mass ratios of the $m_{i}, 1 \leq i \leq k$. However, all of the equations involving the masses $m_{k+1}, \ldots, m_{n}$ vanish identically, so these masses are arbitrary. Therefore the projection map $\pi_{12}: U_{\alpha} \rightarrow V_{k}$ has fibers of dimension $n-k$. But since $\operatorname{dim} V_{k}=k-1$ we still get $\operatorname{dim} \Gamma_{a} \leq k-1+n-k=n-1$. QED

Let $\Gamma_{D} \subset \Gamma$ be the union of all the Dziobek components. For a fixed mass $[m] \in \mathbf{C P}^{n-1}$ define the fiber $\Gamma_{D}([m])=\left\{([r],[z]):([r],[z],[m]) \in \Gamma_{D}\right\}$. Then the following is our main result on generic finiteness.

Corollary. There is a proper subvariety of the mass space, $B \subset \mathbf{C P}^{n-1}$, such that if $[m] \in \mathbf{C P}^{n-1} \backslash B$, the fiber $\Gamma_{D}([m])$ is finite.

Proof. It suffices to consider each of the irreducible components $\Gamma_{\alpha}$ of $\Gamma_{D}$. If $\Gamma_{\alpha}$ is not mass-dominant, then we include the projective closure $\overline{\pi_{3}\left(\Gamma_{\alpha}\right)}$ in the set $B$. Then for $[m] \in \mathbf{C P}^{n-1} \backslash B$, the part of the fiber in $\Gamma_{\alpha}$ is empty.

If $\Gamma_{\alpha}$ is mass-dominant, then by theorem 2 , we have $\operatorname{dim} \Gamma_{\alpha}=n-1$. Since the projection is a regular mapping, the theorem follows from part iii of proposition 4 . QED

Finally, we come back to the realm of real variables and central configurations.

Theorem 3. There is a proper subvariety of the mass space, $B \subset \mathbf{R P}^{n-1}$, such that if $[m] \in \mathbf{R P}^{n-1} \backslash B$, then $[m]$ admits only a finite number of central configurations of dimension $\delta(x)=n-2$ up to symmetry. Moreover, for these masses, the number of configurations is bounded by a number independent of $[\mathrm{m}]$.

Proof. If $B \subset \mathbf{C P}^{n-1}$ is the subvariety of the last theorem, then as we have already observed above, $B \cap \mathbf{R} \mathbf{P}^{n-1}$ is a proper subvariety of $\mathbf{R} \mathbf{P}^{n-1}$. Thus we have the result of the corollary for generic real masses. This implies that for generic real masses there are only finitely many possible values for the mutual distances $r_{i j}$ of a Dziobek configuration, up to scaling. Since the mutual distances determine the configuration up to rotation and reflection symmetry, the first part of the theorem is proved.

For the second part we appeal to the theory of Thom and Milnor on the homology of varieties [14 10]. This provides an upper bound on the number of connected 
components of any real algebraic variety which depends only on the number of variables and the degrees of the equations used to define the variety.

Choose a mass $[m] \in \mathbf{R P}^{n-1} \backslash B$. Equations (20) and (21) are a set of polynomial equations for the mutual distances, $r_{i j}$, and the parameter $r_{0}$ whose degrees are independent of the chosen masses. The inclusion of non-Dziobek components may introduce spurious solutions in addition to those found above, but each of the finitely many solutions we found determines its own component of the total solution set. So the number of these solutions satisfies a mass-independent bound as claimed. QED

\section{REFERENCES}

1. A. Albouy, Symétrie des configurations centrales de quatre corps, C. R. Acad. Sci. Paris 320 (1995), 217-220. MR 95k:70023

2. A. Albouy, Recherches sur le problème des $n$ corps, Notes scientifiques et techniques du Bureau des Longitudes, Paris, (1997) 78.

3. O. Dziobek, Über einen merkwürdigen Fall des Vielkörperproblems, Astron. Nach. 152 (1900) 33-46.

4. J. Harris, Algebraic Geometry, A First Course, Springer-Verlag, Berlin, Heidelberg, New York (1992). MR 93j:14001

5. R. P. Kuz'mina, On an upper bound for the number of central configurations in the planar n-body problem, Soviet Math. Dokl. 18 (1977) 818-821. (Russian orig., MR 58:13169)

6. J.L. Lagrange, Ouvres, vol 6, 272.

7. P. S. Laplace, Sur quelques points du système du monde, Mémoires de l'Académie Royale des Sciences de Paris (1789) article XXIII ou Oeuvres Complètes, vol 11, 553.

8. R. Lehmann-Filhés, Ueber zwei Fälle des Vielkörpersprblems, Astron. Nach. 127 (1891) 137-143.

9. W. D. MacMillan \& W. Bartky, Permanent configurations in the problem of four bodies, Trans. Amer. Math. Soc. 34 (1932) 838-875.

10. J. Milnor, On the Betti numbers of real varieties, Proc. Amer. Math. Soc. 15 (1964) 275-280. MR 28:4547

11. R. Moeckel, Relative equilibria of the four body problem, Erg.Th.Dyn.Sys. 5 (1985) 417-435. MR 87b:70011

12. F.R. Moulton, The straight line solutions of the problem of $N$ bodies, in Ann. of Math. 2-12 (1910) $1-17$.

13. I. R. Shafarevich, Basic Algebraic Geometry 1, Varieties in Projective Space, Springer-Verlag, Berlin, Heidelberg, New York (1994). MR 95m:14001

14. R. Thom, Sur l'homologie des variétés algébriques réelles, in Differential and Combinatorial Topology, Princeton Univ. Press (1965) 255-265. MR 34:828

15. W. L. Williams, Permanent configurations in the problem of five bodies, Trans. Amer. Math. Soc. 44 (1938) 563-579.

16. A. Wintner, The Analytical Foundations of Celestial Mechanics, Princeton Math. Series 5, Princeton University Press, Princeton, NJ (1941). MR 3:215b

School of Mathematics, University of Minnesota, Minneapolis, Minnesota 55455

E-mail address: rick@math.umn.edu 